'Unidad de Cuidados Intensivos, Hospital Británico de Buenos Aires. Argentina.

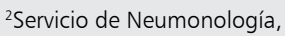
Hospital Británico de Buenos Aires. Argentina.

${ }^{3}$ Servicio de Histopatología, Hospital Británico de Buenos Aires. Argentina.

Este trabajo fue realizado sin financiamiento.

Recibido el 8 de abril de 2016, aceptado el 6 septiembre de 2016.

Correspondencia a: Julio F Chertcoff Hospital Británico de Buenos Aires, Argentina. Perdriel 74, Unidad de Cuidados Intensivos, $2^{\circ}$ piso; CP $1280 \mathrm{AEB}$ TE: (5411) 43096400 int 2808 fchertcoff@yahoo.com.ar

\section{Neumonía intersticial inducida por bloqueo androgénico máximo como tratamiento de cáncer de próstata avanzado}

\author{
GUILLERMO MOLINA MANCERO ${ }^{1, \mathrm{a}}$, XAVIER PICÓN ${ }^{1, \mathrm{a}}$, \\ FERNANDO DI TULLIO ${ }^{2, \mathrm{a}}$, GLENDA ERNST ${ }^{2, \mathrm{~b}}$, PABLO DEZANZO ${ }^{3, \mathrm{a}}$, \\ ALEJANDRO SALVADO ${ }^{2, a}$, JULIO F. CHERTCOFF ${ }^{1,2, a}$
}

\section{Fatal interstitial lung disease associated with maximum androgen blockade. Report of one case}

\begin{abstract}
Maximum androgen blockade is the standard endocrine treatment for advanced prostate cancer. Interstitial lung disease in different degrees of severity, with low mortality and excellent response to treatment may appear with its use. We report a 77 years old patient with advanced prostate cancer who developed severe and progressive respiratory failure associated to bilateral pulmonary infiltrates, attributed to the direct effect of maximum androgen blockade. Despite the therapeutic efforts, the patient died. Lung pathology revealed Usual Interstitial Pneumonia.
\end{abstract}

(Rev Med Chile 2016; 144: 1356-1359)

Key words: Bicalutamide; Idiopathic Pulmonary Fibrosis; Lung Diseases, Interstitial; Respiratory Failure; Respiratory Insufficiency.

\footnotetext{
E
} 1 bloqueo hormonal máximo (BAM) con fármacos antiandrogénicos y análogos de la hormona liberadora de gonadotropinas, tales como bicalutamida y leuprorelina, constituyen en la actualidad el tratamiento endocrinológico estándar para el manejo del cáncer de próstata avanzado. Entre las reacciones adversas severas, se ha informado el desarrollo de enfermedad pulmonar intersticial en un número reducido de pacientes ${ }^{1}$.

Presentamos un caso de enfermedad pulmonar intersticial de evolución subaguda en un paciente con bloqueo androgénico máximo.

\section{Caso clínico}

Hombre de 77 años, ex-fumador de 40 paquetes/año que cesó hace 30 años, antecedentes de hipertensión arterial, hipotiroidismo y bloqueo aurículo-ventricular de tercer grado que requirió marcapasos. Presentó adenocarcinoma semi-diferenciado de próstata con metástasis en pulmón, mediastino y hueso que fue tratado con doble bloqueo hormonal máximo (bicalutamida y leuprorelina) durante el último año. El paciente consultó por progresión de su disnea habitual, de clase funcional (CF) 1 a CF4, de una semana de duración, asociado a tos seca y registros febriles por lo que se interna. En el examen físico destaca apremio respiratorio, frecuencia respiratoria: 25 ciclos/min, frecuencia cardiaca: 96 latidos $/ \mathrm{min}$, presión arterial: 120/70 mm de $\mathrm{Hg}$, temperatura $37^{\circ} \mathrm{C}$ y crepitaciones en ambas bases pulmonares. El laboratorio evidenció insuficiencia respiratoria $\left(\mathrm{PaO}_{2}: 56 \mathrm{~mm}\right.$ de $\mathrm{Hg}$ ), sin hipercapnia, hipercalcemia ( $\mathrm{Ca}: 11,2 \mathrm{mg} / \mathrm{dL})$, aumento de los reactantes 

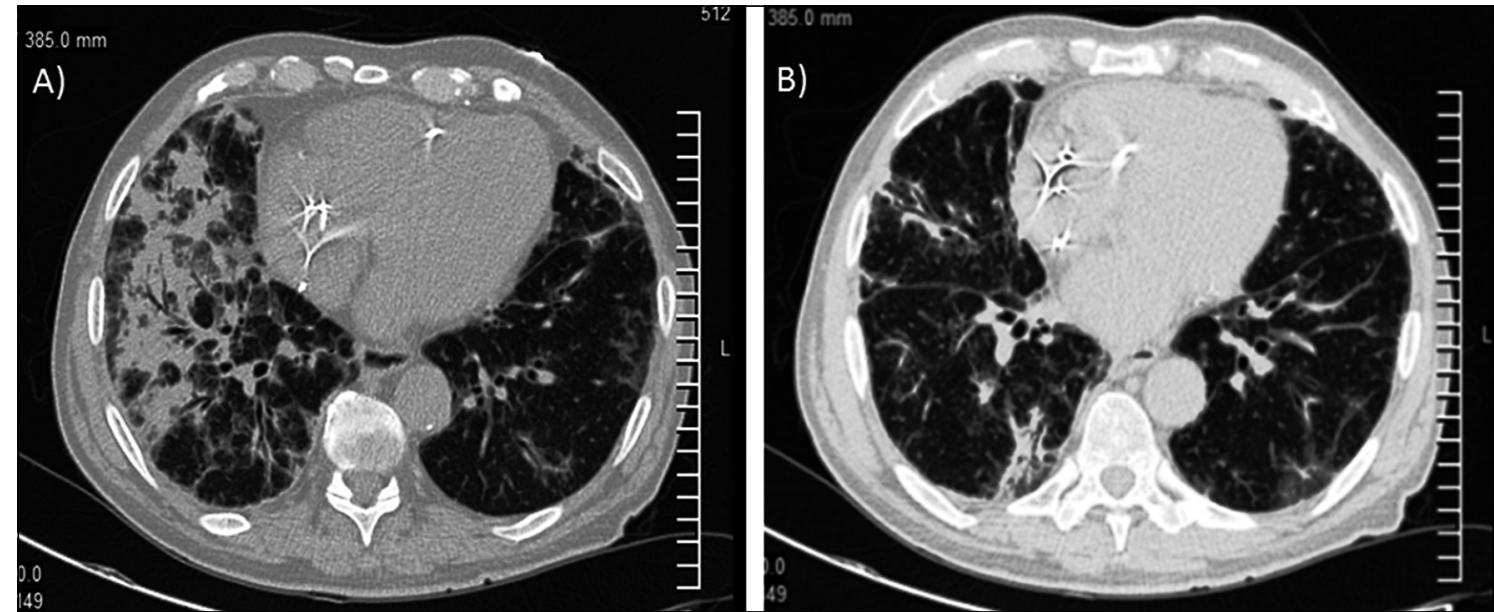

Figura 1. Tomografía de Tórax. Se observa el engrosamiento septal con áreas de vidrio esmerilado y consolidaciones bilaterales con broncograma aéreo en los lóbulos inferiores.

de fase aguda y leucocitosis. La tomografía de tórax mostró consolidaciones bilaterales con predominio en lóbulos inferiores y broncograma aéreo junto con áreas en vidrio esmerilado y engrosamiento septal (Figuras 1 a y b). Se inició tratamiento antibiótico interpretándose el cuadro como neumonía asociada a cuidados de la salud, aunque no se obtuvieron rescates microbiológicos en hemocultivos y urocultivo, no se realiza toma de muestra respiratoria invasiva por inestabilidad hemodinámica. Evoluciona con fibrilación auricular de alta respuesta ventricular (FAARV) y edema agudo de pulmón (EAP), lo que originó su ingresó a unidad coronaria donde inició ventilación no invasiva (VNI) y tratamiento farmacológico: Amiodarona (300 mg vía venosa), beta-bloqueantes y anti-agregación plaquetaria para la patología cardíaca aguda. Ante la falta de respuesta clínica, se realizó cateterismo cardíaco derecho que permitió descartar hipertensión pulmonar. Posteriormente, el paciente ingresó al servicio de Terapia Intensiva donde recibió tratamiento antibiótico empírico de amplio espectro: vancomicina, meropenem, y ventilación no invasiva (VNI).

Debido a la progresión del cuadro de insuficiencia respiratoria y falta de respuesta a la VNI requirió intubación orotraqueal, conexión a ventilación mecánica e inicio de drogas vasopresoras. Evoluciona sin mejoría a pesar del tratamiento instaurado, se sospechó en este contexto de neumonía organizada, lo que motivó el tratamiento con metilprednisolona $1 \mathrm{~g} /$ día por cinco días que luego continuó con prednisona $60 \mathrm{mg} /$ día.

El cuadro clínico progresó a hipoxemia refractaria y continuó con evolución desfavorable produciéndose el deceso.

El tiempo de estadía hospitalaria fue de un total de 21 días de los cuales cinco correspondieron a internación en sala general, 9 en Unidad coronaria y los últimos 7 días en Unidad de Terapia Intensiva.

El resultado de la anatomía patológica de la muestra pulmonar post-mortem reveló la presencia de neumonía en organización $(\mathrm{OP})$ y neumonía intersticial usual (UIP) (Figuras $2 \mathrm{a}$ y b).

\section{Discusión}

El adenocarcinoma es el tipo más común del cáncer de próstata; la mediana de edad al momento del diagnóstico es de 66 años $^{2}$. En estadios avanzados, el bloqueo androgénico máximo, es el tratamiento endócrino estándar para el cáncer de próstata. Bicalutamida es un anti-andrógeno no esteroidal que se usa como parte del BAM con mínima toxicidad ${ }^{3}$. Las reacciones adversas más frecuentes son: dolor pélvico, astenia, constipación, diarrea y hematuria, aunque también han sido reportados pacientes que desarrollaron enfermedad intersticial pulmonar luego de iniciar el tratamiento ${ }^{4-6}$. El primer caso reportado de 


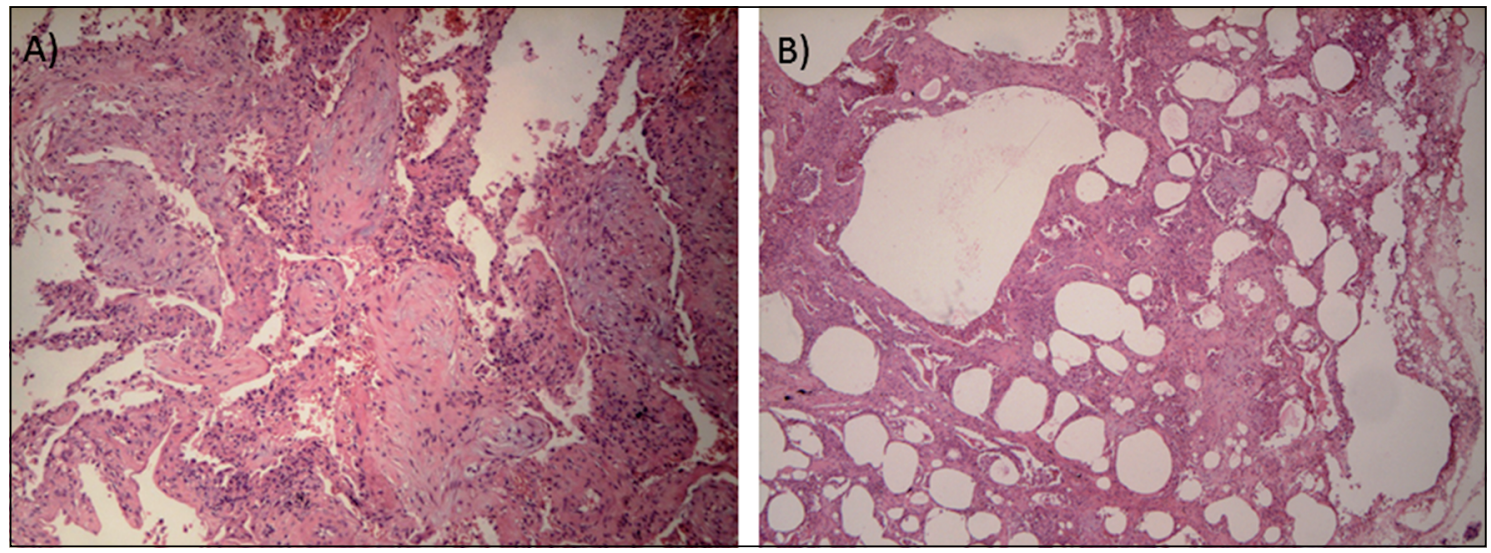

Figura 2. Biopsia Pulmonar. A) Se observa taponamiento fibroconectivo de vías aéreas distales (cuerpos de Masson) H\&E 100x. B) Patrón de compromiso en parches. H\&E 40x.

toxicidad pulmonar por bicalutamida se remonta a $1998^{3}$.

El tratamiento antiandrogénico con bicalutamida se ha asociado a neumonía intersticial subaguda $^{4-6}$ y neumonía eosinofílica ${ }^{7}$; mientras que la leuprorelina se ha asociado a neumonía intersticial subaguda, síndrome de dificultad respiratoria aguda (ARDS), derrame pleural sanguinolento y miositis $^{6,8-13}$.

Se ha descrito que la neumonía intersticial inducida por los fármacos anti-andrógenos no esteroidales como bicalutamida, seinicia por lo general a partir de los 2-8 meses de uso y que estos efectos se presentan especialmente con dosis mayores a $50 \mathrm{mg} / \mathrm{día}^{5}$. Los agonistas de la hormona luteinizante-hormona reguladora de gonadotrofina como leuprolide también ocasionan neumonía intersticial ${ }^{3,5}$. Algunos autores han sugerido que la toxicidad pulmonar inducida por estas drogas podría estar relacionada con un efecto directo de las mismas o por un mecanismo de hipersensibilidad ${ }^{5,6}$. Se considera por tanto a la fibrosis pulmonar inducida por bicalutamida una condición extremadamente rara pero severa $y$ a veces fatal a pesar del tratamiento instaurado ${ }^{8}$.

En el caso de nuestro paciente, todos los factores predisponentes antes descritos estuvieron presentes: varón mayor de 65 años, con BAM mayor a ocho meses de duración con dosis de bicalutamida mayor a $50 \mathrm{mg} /$ día y administración concomitante de leuprolide. Las imágenes radiológicas son com- patibles, la ausencia de microorganismos aislados en las diferentes muestras biológicas asociado ala mala respuesta al tratamiento antibiótico, reforzaron el diagnóstico.

A pesar de los esfuerzos realizados el paciente falleció. Si bien algunos casos presentados en la literatura, mostraron una mejoría clínica rápida con la retirada de bicalutamida-leuprolide más corticoides sistémicos alcanzando buen pronóstico en el evento agudo, en otros casos la progresión de la enfermedad pulmonar condujo a la muerte que fue atribuida al episodio de fibrosis pulmonar ${ }^{8,9}$. Para concluir, en el caso descrito, la anatomía patológica post-mortem fue determinante. Es el segundo caso reportado que muestra elementos de neumonía en organización $(\mathrm{OP})^{3}$.

\section{Referencias}

1. Pfitzenmeyer P, Foucher P, Piard F, Coudert B, Braud ML, Gabez P, et al. Nilutamide pneumonitis: a report on eight patients. Thorax 1992; 47: 622-7.

2. Zhou CK, Check DP, Lortet-Tieulent J, Laversanne M, Jemal A, Ferlay J, et al. Prostate cancer incidence in 43 populations worldwide: An analysis of time trends overall and by age group.Int J Cancer 2016; 138: 1388-400.

3. McCaffrey JA, Scher HI. Interstitial pneumonitis following bicalutamide treatment for prostate cancer. J Urol 1998; 160 (1): 131.

4. Highlilights of prescribing information. CASODEX ${ }^{\circledR}$. Full prescribing information. Food and Drug Adminis- 
tration (FDA). Disponible en: http://www.accessdata. fda.gov. Último acceso 09/01/2016.

5. Masago T, Watanabe T, Nemoto R, Motoda K. Interstitial pneumonitis induced by bicalutamide given for prostate cancer. Int J Clin Oncol 2011; 16: 763-5.

6. Shioi K, Yoshida M, Sakai N. Interstitial pneumonitis induced by bicalutamide and leuprorelin acetate for prostate cancer. Int J Urol 2003; 10: 625-6.

7. Wong PW, Macris N, DiFabrizio L, Seriff NS. Eosinophilic lung disease induced by bicalutamide: a case report and review of the medical literature. Chest 1998; 113: 548-50.

8. Azuma T, Kurimoto S, Mikami K, Oshi M. Interstitial pneumonitis related to leuprorelin acetate and flutamide. J Urol 1999; 161 (1): 221.

9. Shioi K, Sakai N, Yoshida M, Nakamura M. Successful recovery from interstitial pneumonitis, induced by bi- calutamide and leuprorelin acetate given as treatment for prostate cancer. Hinyokika Kiyo 2005; 51: 211-4.

10. Crayton H, Bohlmann T, Sufit R, Graziano FM. Drug induced polymyositis secondary to leuprolide acetate (Lupron) therapy for prostate carcinoma. Clin Exp Rheumatol 1991; 9: 525-8.

11. Gamelin L, Jeanson S, Chenue F, Gouello JP, Lainé-Cessac P, Bouachour G, et al. Acute respiratory distress syndrome after injection of leuprorelin Therapie 1995; 50: 587-8.

12. Wieder JA, Soloway MS. Interstitial pneumonitis associated with neoadjuvant leuprolide and nilutamide for prostate cancer. J Urol 1998; 159 (6): 2099.

13. Bergner M, Rohacek M, Erne P. Inflammatory myopathy and severe rhabdomyolysis induced by leuprolide acetate therapy for prostate cancer: a case report. J Med Case Rep 2011; 5: 409. 